“C 2011 IEEE. Personal use of this material is permitted. Permission from IEEE must be obtained for all other uses, in any current or future media, including reprinting/republishing this material for advertising or promotional purposes, creating new collective works, for resale or redistribution to servers or lists, or reuse of any copyrighted component of this work in other works." 


\title{
Robust Multilevel Optimization of PMSM Using Design for Six Sigma
}

\author{
Xiangjun Meng ${ }^{1,2}$,Shuhong Wang ${ }^{1}$, Jie Qiu ${ }^{1}$, Qiuhui Zhang ${ }^{1}$, Jian Guo Zhu ${ }^{3}$, Youguang Guo ${ }^{3}$ \\ ${ }^{1}$ State Key Laboratory of Electrical Insulation and Power Equipment, Faculty of Electrical Engineering, Xi'an Jiaotong \\ University, 28 West Xianning Road, Xi'an, 710049, China \\ shwang@mail.xjtu.edu.cn \\ ${ }^{2}$ XJ Flexible Transmission System Corporation, Electric city, Xuchang, 461000, China \\ ${ }^{3}$ Faculty of Engineering and Information Technology, University of Technology, Sydney, NSW2007, Australia
}

\begin{abstract}
In this paper, a robust optimization method based on Design for Six Sigma (DFSS) is combined to the optimization of a surface mounted permanent synchronous machine (PMSM) by using Multilevel Genetic Algorithm (MLGA). Firstly, MLGA and DFSS are introduced in the robust optimization. Secondly, by taking into account the tolerances of the motor production, important input parameters could be varied with six sigma distribution and monte-carlo simulation (MCS) method is used to reduce the calculation cost. Thirdly, to verify the new algorithm, the presented algorithm is applied to the optimization of a PMSM. The results compared with those of traditional GA and MLGA and the discussion of the robust optimization combined with MLGA are presented.
\end{abstract}

Index Terms-Multilevel Genetic algorithm, robust optimization, design for six sigma, permanent magnet (PM) machine

\section{INTRODUCTION}

$\mathrm{N}_{\mathrm{n}}$ umerical simulation technology and optimization method have been applied to improve the design quality and shorten the design cycle of the PMSM. However, the existence of fluctuation in design variables or operation conditions has a great influence on the motor properties.

DFSS is an effective method to improve the design quality and decrease the sensitive of product quality to uncertain. Y. Q. Li [1] employs the six design method to the optimization of deep-drawing sheet metal process combined with the dual response surface model and design of experiment; the optimal results improve the reliability and robustness of the production and also increase the design efficiency. P.N. Koch [2] presents an implementation of design for six sigma to measure the design quality of the production through mathematical method. X.Y. Liu [3] used DFSS to the optimization of current distribution among the multilayer conductors in a high-temperature superconducting (HTS) cable.

In order to estimate the effects of parameter perturbations in design and to improve the design efficiency, a robust optimization method based on design for six sigma (DFSS) is presented in this paper. The optimization results shows that the proposed optimization procedure can not only achieve a better performance of motor, but also improve significantly the reliability and robustness of the PMSM performance, comparing with those by using GA and multilevel Genetic Algorithm.

\section{MultileVel Genetic Algorithm}

Multilevel optimization is described by using the problem matrix which may be used to allocate the design variables on different levels. And the parameters in the problem matrix are deduced by using correlation analysis [4]. The architecture and implementation of multilevel genetic algorithm are carried out base on multilevel optimization. The architecture of MLGA is shown in Fig. 1. In MLGA the design optimization variables are classified and allocated to different levels according to the relative importance among the variables and objective functions, constraints, as well as the practical engineering weight and optimization sequence. The variables on different levels are encoded independently. Each level may have multiple populations and each of them can adopt different dynamic genetic operators and parameters. Furthermore, the relationship between sub-problems in multilevel problems can be handled by MLGA.

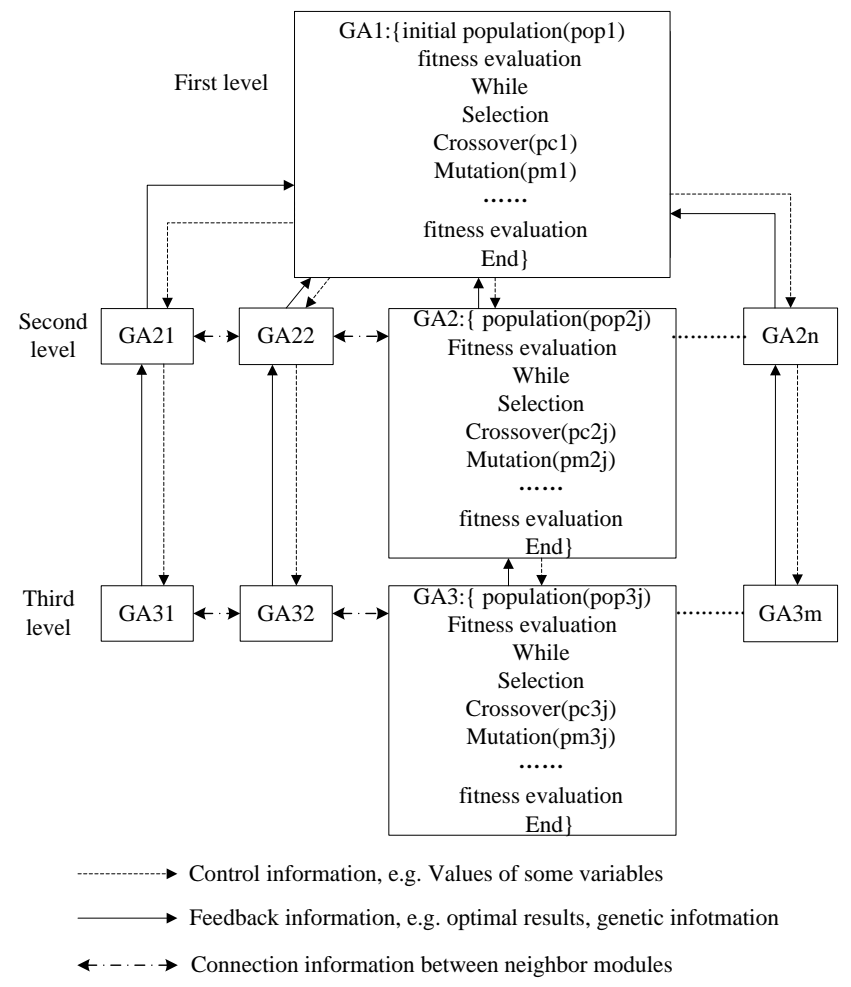

Fig. 1. Block diagram of MLGA 
In Fig.1, the $\mathrm{GA}_{1}$ is the master GA module and $\mathrm{GA}_{2 \mathrm{i}}$, $\mathrm{GA}_{3 \mathrm{i}}$ consist of a number of modules, in which each module corresponds to a sub-system. The sub-system in the multilevel structure is not independent for the interactions between the sub-systems on upper and lower levels. The module in the upper level of the MLGA acts as a solver which affects GA of other sub-system.

\section{The implementation process of MLGA is as follows.}

First, determine the objective functions, constraints and design variables. Second, make analysis using correlation analysis, then determine the architecture of MLGA. Third, allocate all the requirements and build up the relationships among different levels and different modules on each level. Each module corresponds to a genetic algorithm module. Forth, implement MLGA and feedback messages. Last, reach the termination criterion and end the total solving process.

MLGA possesses some special advantages as follows.

The encoding of design variables on lower level chromosome may be modified with upper level chromosome. The diversity of the population can be independently enhanced by the parallel genetic operations performed in different modules within one level. The genetic operators of selection, crossover, mutation, population size and number of evolution generations can dynamically change in the implementation for each independent module.

For optimization of SPMSM, sometimes, MLGA may save the finite element (FE) calculation time. For example, on the basis of given structural and material parameters, select the thickness and width of permanent magnets as design variables on Level 1 and assign the conductor number per slot and diameter of the conductors as design variables on Level 2. On level 1, calculate d-axis and q-axis components of inductances per turn by the no-load EMF when design variables are modified. On other levels, the thickness and width of permanent magnets are determined on Level 1, the EMF, d-axis and q-axis components of inductances are proportional to the conductors per slot which means FEM will not be conducted on Level 2. The computing cost of FEM in MLGA is less than that in traditional GA for the total of populations and evolution generations of traditional GA are equal to those of MLGA,

\section{DFSS ROBUST OPTIMIZATION APPROACH}

The six-sigma methodology was proposed at Motolola and developed into DFSS at General Electric (GE). DFSS is one of the robust optimization methods, and the term "sigma" here refers to standard deviation $\sigma$, which is a measure of dispersion. The performance level $6 \sigma$ is equivalent to 3.4 defect parts per million (PPM), while at $3 \sigma$ level (the average sigma level for most companied) the defect ratio is about 66800 PPM.
During traditional optimization problem, the objective function $f(\boldsymbol{X})$ of design variable $\boldsymbol{X}$ should be minimized or maximized and subjected to constraints $g_{k}(\boldsymbol{X})$ as follows. $\min f(X)$

s.t. $g_{k}(X) \geq 0, \quad k=1,2, \ldots$, Number of constraints

In DFSS, six sigma and reliability are combined to define the robustness of disturbance, constraints and the original object function and constraints may be rewritten as

$$
\begin{cases}\text { Min } & F\left(\mu_{f}(X), \sigma_{f}(X)\right) \\ \text { s.t. } & g_{i}\left(\mu_{f}(X), \sigma_{f}(X)\right) \leq 0 \\ & X_{L}+n \sigma_{X} \leq \mu_{X} \leq X_{U}-n \sigma_{X} \\ & \mu_{f}-n \sigma_{f} \geq L S L \\ & \mu_{f}+n \sigma_{f} \leq U S L\end{cases}
$$

where, $L S L, U S L, \mu_{f}$ and $\sigma_{f}$ are the lower bound, upper bound, mean value and standard deviation of the original function, respectively, $X$ is the input design variables, $X_{L}$, $X_{U}, \mu_{X}, \sigma_{X}$ are the lower bound, upper bound mean value and standard deviation of the variables, respectively. $n$ is the sigma level.

Robust optimization should consider the mean value and minimum variation of the objective function, it can be defined as

$$
F=\sum_{i=1}^{l}\left[\frac{\omega_{1 i}}{s_{1 i}}\left(\mu_{f i}-M_{i}\right)^{2}+\frac{\omega_{2 i}}{s_{2 i}} \sigma_{f i}^{2}\right]
$$

where, $\omega_{1 i}, \omega_{2 i}$ are the weight factors to mean and minimum variation to the objective function. $s_{1 i}, s_{2 i}$ are the proportion factors to mean and minimum variation of the objective function. $M_{i}$ is the mathematical expectation and $l$ is the number of performances concerned.

\section{Robust OPtimization Model OF PMSM}

In order to verify the proposed methodology, a PMSM controlled by field oriented control (FOC), rated at $1000 \mathrm{~W}$ output power, $2000 \mathrm{rpm}$ speed and $128 \mathrm{~V}$ line to line voltage is used to verify the MLGA and DFSS based robust optimization.

The bi-level optimization model is defined as follows

$$
\begin{aligned}
& \max f_{1-2}\left(X_{1}\right)=K /\left(\omega_{1} \frac{\cos t(\mathrm{Cu})}{\max (\mathrm{Cu})}+\omega_{2} \frac{\cos t(\mathrm{PM})}{\max (\mathrm{PM})}+\omega_{3} \frac{100-\eta}{100}\right) \\
& \text { s.t. } s f<0.78 \\
& \qquad p_{2}>745 \mathrm{~W}
\end{aligned}
$$

where, design variable $X_{1}=[\mathrm{hm} b m$ Ns WindD $]$; $\max (\mathrm{Cu})$ and $\max (\mathrm{PM})$ are possible maximum of the cost of stator windings and permanent magnets, respectively; $\operatorname{cost}(\mathrm{Cu})$ and $\operatorname{cost}(\mathrm{PM})$ represent the cost of stator windings and magnets, respectively; $\eta$ is the efficiency of SPMSM, $K, \omega_{1}$, $\omega_{2}$ and $\omega_{3}$ are weight factors defined by designer. $P_{2}$ is 
output power and $s f$ is fill factor, $h m$ and $b m$ are thickness and width of the permanent magnet. $N s$ and WindD are conductors per slot and the conductor diameter, and $\mathrm{hm}, \mathrm{bm}$ and WindD are selected as robust optimization variables.

According to (2) and (3), (4) is modified as

$$
\begin{aligned}
& \text { Minimize }: F=\omega_{\mu}\left[\mu\left(f_{1-2}(X)\right)-M\left(f_{1-2}(X)\right)\right]^{2}+\omega_{\sigma} \sigma\left(f_{1-2}(X)\right)^{2} \\
& \text { subject to }: X_{L}+n \sigma_{X} \leq \mu_{X} \leq X_{U}-n \sigma_{X} \\
& \mu_{s f}-n \sigma_{s f}<0.78 \\
& \mu_{p_{2}}-n \sigma_{p_{2}}>745
\end{aligned}
$$

In the bi-level optimization, $\mathrm{hm}$ and $\mathrm{bm}$ are assigned on Level 1 and WindD are allocated on Level 2, respectively. The robust optimization model described in (5) is regarded as the common objective function and constraints on both Level 1 and 2.

In this paper, Monto-Carlo Simulation (MCS) method is used to implement the random simulation sampling, to avoiding large-scale sampling, the descriptive sampling method is employed. Data around the design variables are sampled and calculated to get the objective values. The reliability and robust of the design variables are evaluated according to those sample points values.

The MCS can be described as follows:

First, the design variables are sampled in the disturbance scale by using regular sampling method according to the step length.

Second, the sample points set is rearranged according to the actual probability distribution [2]. The reliability and robust of the design variables are evaluated by (5).

This paper combines the DFSS with the MLGA optimization of PMSM. The flowchart is shown in Fig. 2.

\section{RESULTS}

Table I lists the robust optimization results when given different design variables disturbance scale and weight factors $\omega_{\mu}$ and $\omega_{\sigma}$ in the objective function (5). The sigma level is set as $n=3$, and the number of sample points is 10 .

The fitness of the objective function varies with the disturbance of the variables, the bigger change range of the fitness, the bigger deviation degree of the objective function That is the lower robust of the system. When the disturbance scale is \pm 0.05 and the number of sample points is 100 , we will get the frequency histogram of the objective function. Fig.3 and Fig. 4 are DFSS and MLGA optimization frequency histogram, respectively. It can be seen from those two figures when DFSS is used to the robust optimization, the range of the objective function is $0.266 \sim 0.274$, and MLGA is $0.23 \sim 0.26$. The latter range of MLGA is 0.022 , which is bigger than that of DFSS. It is suggested that the robustness of the robust optimization is higher than MLGA optimization.

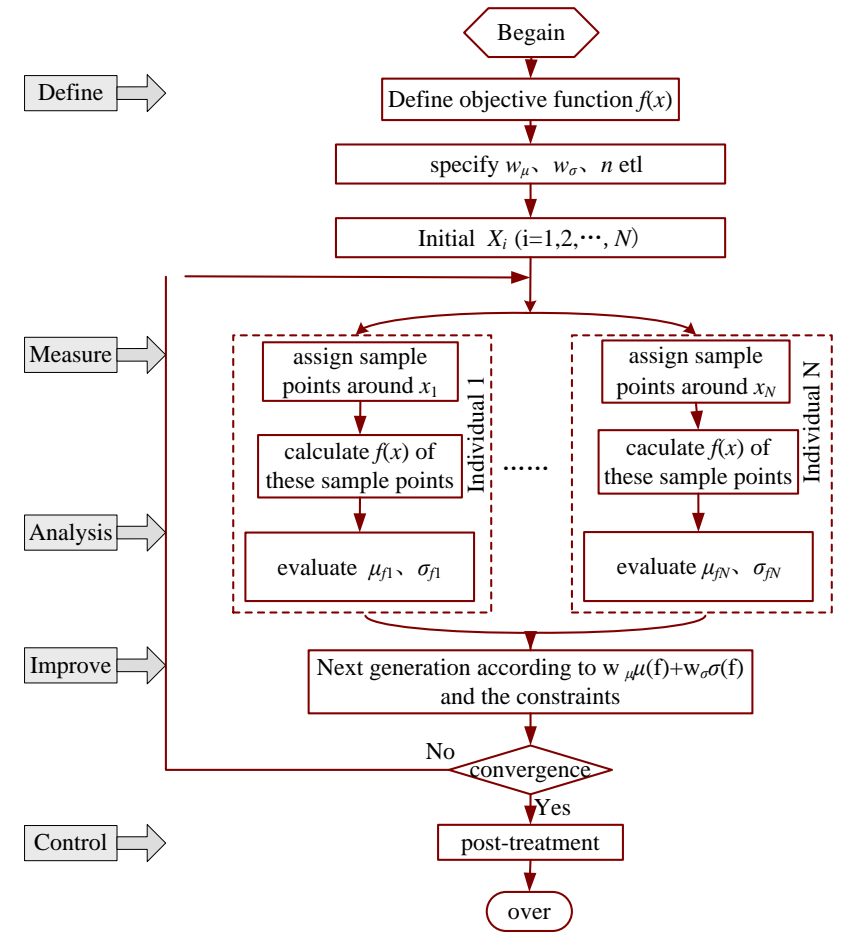

Fig. 2. Flowchart of DFSS based MLGA

TABLE I

ROBUST OPTIMIZATION RESULT OF PMSM

\begin{tabular}{ccccc}
\hline \hline $\begin{array}{c}\text { Disturbance } \\
\text { scale }\end{array}$ & $\omega_{\mu}: \omega_{\sigma}$ & Fitness & $\mu_{f}$ & $\sigma_{f}$ \\
\hline \pm 0.01 & $1: 100$ & 2.1330 & 0.2120 & $1.2097 \mathrm{e}-006$ \\
\pm 0.01 & $1: 50$ & 1.8147 & 0.2009 & $8.4983 \mathrm{e}-007$ \\
\pm 0.01 & $1: 10$ & 2.0520 & 0.1999 & $2.9441 \mathrm{e}-007$ \\
\pm 0.025 & $1: 50$ & 2.2769 & 0.2359 & $1.2210 \mathrm{e}-006$ \\
\pm 0.05 & $1: 50$ & 2.0403 & 0.1979 & $2.4259 \mathrm{e}-006$ \\
\hline \hline
\end{tabular}

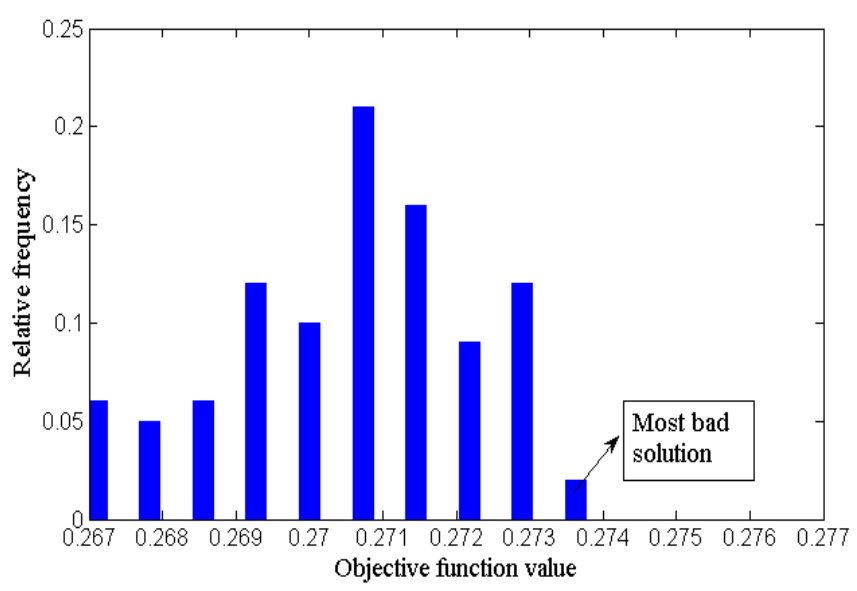

Fig. 3. DFSS optimization frequency histogram 


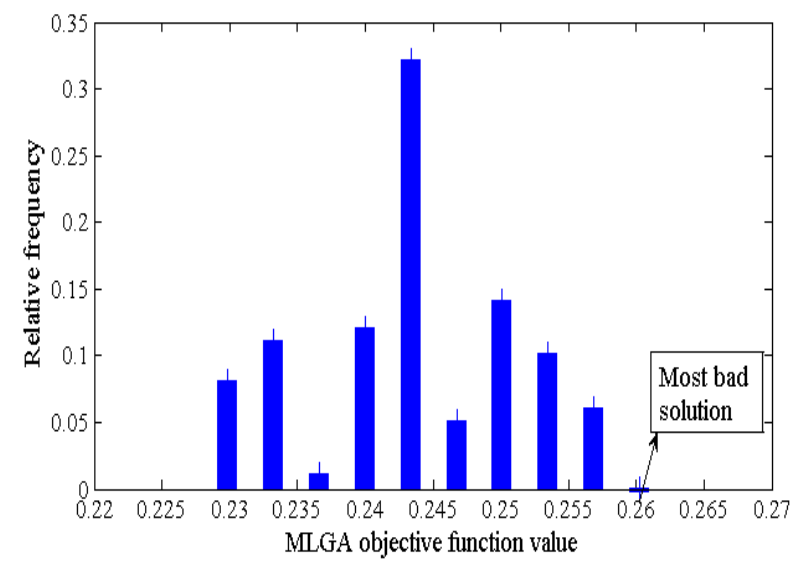

Fig. 4. MLGA optimization frequency histogram

Table II lists the optimization results for PMSM by using MLGA and DFSS respectively. Both optimization methods may provide better performances than that of original design. Although the efficiency achieved by MLGA is little higher than that of DFSS, the cost of windings and permanent magnets optimized by DFSS are less than that calculated by using MLGA. It is crucial that the results optimized by DFSS possesses higher reliability than those analyzed by MLGA.

TABLE II

RESULTS COMPARISON OF MLGA AND DFSS

\begin{tabular}{cccc}
\hline \hline Variables and performance & Original & MLGA & DFSS \\
\hline Thickness of PM, $h m / \mathrm{cm}$ & 0.18 & 0.23 & 0.1788 \\
Width of PM, $b m / \mathrm{cm}$ & 3.14 & 3.03 & 3.0018 \\
Conductors per slot, $N s$ & 72 & 67 & 83 \\
Diameter of conductor, WinD / mm & 0.5 & 0.56 & 0.50 \\
Back-EMF EO / V & 66.0 & 61.9 & 75.7777 \\
q-axis component of current $I q$ / A & 4.78 & 5.27 & 4.3258 \\
d-axis component of current $I d /$ A & 1.60 & 0.05 & 0.0503 \\
Efficiency, $\eta(\%)$ & 83.7 & 86.4 & 85.7811 \\
Cost of Wire / RMB & 72.6 & 84.7 & 82.1443 \\
Cost of PM / RMB & 41.3 & 50.9 & 39.3848 \\
Output power, $P_{2} / \mathrm{W}$ & 946 & 949.5 & 951.9331 \\
Fill factor, $s f(\%)$ & 67 & 77.7 & 76.7050 \\
\hline \hline
\end{tabular}

\section{CONCLUSION}

In this paper, considering the uncertainties in PMSM structural design, an optimization algorithm based on design for six sigma combining with MLGA is applied to perform a robust design. The comparison among traditional GA and MLGA and DFSS-based MLGA shows that the robust optimization using DFSS is superior to the MLGA algorithm to achieve a higher reliability and quality.

\section{REFERENCES}

[1] Y. Q. Li, Z. S. Cui, X. Y. Ruan, D. J. Zhang, "CAE-based six sigma robust optimization for deep-drawing sheet metal process," Int J Adv Manuf Technol, vol. 30, 2006, pp: 631-637.

[2] P.N. Koch, R.-J. Yang, L. Gu , "Design for six sigma through robust optimization.," Struct Multidisc Optim, vol. 26, 2004, pp: 235-248.

[3] Xinying Liu, Shuhong Wang, Jie Qiu, Jian Guo Zhu, Youguang Guo, Zhi Wei Lin, "Robust Optimization in HTS Cables Based on Design for Six Sigma," IEEE TRANSACTIONS ON MAGNETICS, vol. 44, no. 6, 2008, pp: 978-981.

[4] Xiangjun Meng, Shuhong Wang, Jie Qiu, Jian Guo Zhu, Yi Wang, Youguang Guo, Dikai Liu, Wei Xu, "Dynamic Multilevel Optimization of Machine Design and Control Parameters for PMSM Drive System Based on Correlation Analysis," IEEE TRANSACTIONS ON MAGNETICS, vol. 46, no. 8, 2010, pp: 27792782 . 